\title{
Parlament und Regierung - Getriebene der Finanzmärkte? Eine Veranstaltung der DVParl am 23. November 2011 in Berlin
}

Im Vorfeld der Abendveranstaltung zum Thema „Parlament und Regierung - Getriebene der Finanzmärkte?" fand am 23. November 2011 die Jahresversammlung der Deutschen Vereinigung für Parlamentsfragen (DVParl) in Berlin statt, auf der ein neuer Vorstand zu wählen war: Vorsitzende wird künftig die Bundestagsabgeordnete Brigitte Zypries, Bundesjustizministerin a.D., sein. Die Justiziarin der SPD-Bundestagsfraktion löst den langjährigen Vorsitzenden Joachim Hörster (CDU) ab. Zu Stellvertretern wurden Heinrich Oberreuter (Emeritus Universität Passau und Akademie für Politische Bildung Tutzing), Georg Paul Hefty (Redakteur der Frankfurter Allgemeinen Zeitung) und Andrea Voßhoff (Vorsitzende der Arbeitsgruppe Recht der CDU/CSU-Bundestagsfraktion) gewählt.

Im Anschluss beleuchtete die DVParl das Verhältnis zwischen Politik und Finanzmärkten. Im Mittelpunkt stand die Frage, ob die politischen Entscheidungsträger, wie es Jürgen Habermas in der FAZ vom 5. November 2011 formulierte, ,an den Drähten der Finanzindustrie zappeln" würden. Zur Beantwortung eingeladen waren der frühere Staatssekretär und Wirtschaftspolitiker Johann Eekhoff sowie Michael Kemmer, Hauptgeschäftsführer und Vorstandsmitglied des Bundesverbandes deutscher Banken. Heinrich Oberreuter moderierte die Veranstaltung. ${ }^{1}$

Gleich zu Beginn seiner Ausführungen brachte Michael Kemmer seine Position auf den Punkt, dass die Politik an Autonomie eingebüßt habe. Verkürzt dargestellt, zwingen die Ereignisse an den Finanzmärkten Parlamente und Regierungen in immer schnellerem Rhythmus Entscheidungen auf, die sie eigentlich nicht treffen möchten und die - schlimmer noch häufig wirkungslos bleiben. Ein Grundproblem stellt die Ungleichzeitigkeit von politischem und Markthandeln dar: Global agierende Finanzmarktteilnehmer verstehen nur schlecht, dass politische Entscheidungen in einer Demokratie stets Kompromisse sind, die Zeit benötigen; sie verlangen dagegen Beschlüsse ohne Kompromisse mit sofortiger Wirksamkeit. Am schwersten dürfte aber wiegen, dass die Wähler die Anstrengungen der Politik nur selten als „notwendige Maßnahmen zur Stabilisierung des gesamten Wirtschaftssystems inmitten einer bedrohlichen Krise" honorieren, sondern vielmehr glauben, dass es sich um Klientelpolitik handele. Das Resultat: Die politischen Entscheidungsträger sind einem Vertrauensverlust ausgesetzt, da viele Bürger ihre Interessen nicht mehr durch die Politik vertreten sehen.

Kemmer unterstrich zugleich, dass die Kausalität in unzulässiger Weise verdreht werde, wenn „der Autonomieverlust der Politik als von global wirtschaftenden Unternehmen oder von den Finanzmärkten oktroyiert" bezeichnet werde. Unbestritten haben Banken und andere Finanzmarktteilnehmer einen erheblichen Anteil an der Entstehung der Finanzkrise: Erstens hat sich die Bewertung der Risiken - abgekoppelt von der Realität - in Teilen zu sehr auf mathematisch-statistische Verfahren verlassen; zweitens waren durch falsche Leistungsanreize für Mitarbeiter und Führungskräfte, kurzfristige Gewinne häufig attraktiver als der langfristige Erfolg; und drittens haben nicht alle Finanzinnovationen den Markttest bestanden. Es sind jedoch auch von den Aufsichtsbehörden und der Politik Fehler gemacht

1 Vgl. auch Jörg Biallas, Viele Antworten - und trotzdem bleiben Fragen. Die Deutsche Vereinigung für Parlamentsfragen beleuchtet das Verhältnis zwischen Politik und Finanzmärkten, in: Das Parlament vom 28. November 2011, S. 11. 
worden. „Eine stabile Weltwirtschaft braucht strukturelle Reformen, die sie insgesamt widerstandsfähiger gegen makroökonomische Schocks macht“, sagte Kemmer und nannte als Ansatzpunkte den Arbeitsmarkt sowie das Renten- und Gesundheitssystem. Er erinnerte daran, dass die Liberalisierung der Märkte „einen erheblichen Wohlfahrtseffekt" gebracht habe. Allerdings seien die Zeiten des mit Schulden finanzierten Wohlfahrtstaates vorüber - in Europa, aber auch in den USA. Da die Schulden von den Staaten und nicht von den Banken gemacht wurden, müssen „die Regierungen akzeptieren, dass der politische Handlungsdruck auch verfehlten politischen Entscheidungen in der Vergangenheit entstammt, also zu einem gewissen Teil selbst erzeugt ist", schlussfolgerte Kemmer.

Der gleichen Ansicht war Johann Eekhoff. Er verwies aber auch auf die Mechanismen der Finanzmärkte, die von Regierungen und Parlamenten nicht immer ausreichend zur Kenntnis genommen würden: „Die Krise in den USA ist entstanden, weil der Staat mehr leisten wollte, als er sich leisten konnte." Laut Eekhoff sei es nicht Aufgabe der Regierung, dafür zu sorgen, dass möglichst jeder Bürger ein eigenes Haus besitzt. Hemmungslose Staatsverschuldung sei besonders gefährlich, wenn das Geld nicht für Investitionen verwendet werde. In diesem Zusammenhang wandte sich Eekhoff energisch gegen einen Rettungsschirm für schwache Euro-Staaten. Damit werde signalisiert, dass gar nicht erwartet wird, dass Länder wie Italien oder Spanien ihre Schulden tatsächlich begleichen müssen. Aus diesem Grund sollte der umgekehrte Weg eingeschlagen werden, indem sich Staaten wieder an eine ganz einfache Regel halten, die für jeden Bürger gilt: „Wer Schulden aufnimmt, zahlt sie zurück." Werde dieses Prinzip ausgehöhlt, sei es ausgeschlossen, eine Währungsunion in Europa dauerhaft aufrecht zu erhalten. Folgt man Eekhoff, ist es vor allem Aufgabe des Staates, dafür zu sorgen, Arbeitslosigkeit abzubauen und damit die Steuereinnahmen zu erhöhen. Dies gilt umso mehr, als eine Entwicklung droht, die im Augenblick noch gar nicht diskutiert wird: die implizite Verschuldung der Sozialversicherungssysteme bei sich verringernder Bevölkerungszahl oder alternder Bevölkerung.

In der anschließenden Diskussion wurden grundsätzliche Fragen aufgeworfen. Josef Isensee (Emeritus Rheinische Friedrich-Wilhelms-Universität Bonn) attestierte der parlamentarischen Demokratie eine indirekte Mitschuld an der Verschuldungskrise: „Die Staatsverschuldung ist [im Wettstreit der Parteien um Wählerstimmen] das Opium des Volkes. Es lässt sich gern süchtig machen, und es ist süchtig gemacht. Die Frage der Rückzahlung stellt sich überhaupt nicht." Kemmer und Eekhoff pflichteten der Sichtweise bei, nicht jedoch ohne darauf hinzuweisen, dass es den Bürgern schwer zu vermitteln sei, dass an der Quelle der Staatsverschuldung ihr eigenes Anspruchsdenken sitzt. Sein Schlusswort begann Oberreuter mit Bertolt Brecht: „Der Vorhang zu und alle Fragen offen.“ Für das Publikum bestand jedoch kein Anlass zur Enttäuschung, hatte es doch in den zwei Stunden zuvor Kenntnisse und Einsichten hinzugewinnen können. Dass die Diskutanten die Fragen, ob, wie und warum die Finanzmärkte die Politik vor sich hertreiben, nicht in allen Facetten abschließend beantworten würden, war angesichts der Komplexität des Themas auch nicht zu erwarten. Oberreuter gab eine eigene Antwort auf die Ausgangsfrage des Abends: „Parlamentarier sind Getriebene der Anspruchshaltung ihrer Wähler", sie sollten sich zumindest nicht ausschließlich von den Erwartungen derer leiten lassen, die sie ins Amt gebracht haben. ${ }^{2}$

Alexander Kühne

2 Vgl. ebenda. 